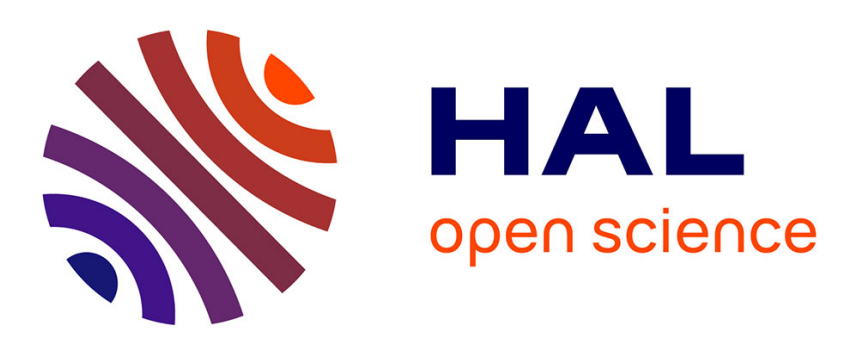

\title{
Experimental thermomechanic study of Newtonian and non-Newtonian suspension flows
}

Michel Gradeck, Benoit Fanou Zinsou Fagla, Christophe Baravian, Michel

Lebouché

\section{To cite this version:}

Michel Gradeck, Benoit Fanou Zinsou Fagla, Christophe Baravian, Michel Lebouché. Experimental thermomechanic study of Newtonian and non-Newtonian suspension flows. International Journal of Heat and Mass Transfer, 2005, 48 (16), pp.3469-3477. 10.1016/j.ijheatmasstransfer.2004.12.052 . hal-01579179

\section{HAL Id: hal-01579179 \\ https://hal.univ-lorraine.fr/hal-01579179}

Submitted on 30 Aug 2017

HAL is a multi-disciplinary open access archive for the deposit and dissemination of scientific research documents, whether they are published or not. The documents may come from teaching and research institutions in France or abroad, or from public or private research centers.
L'archive ouverte pluridisciplinaire HAL, est destinée au dépôt et à la diffusion de documents scientifiques de niveau recherche, publiés ou non, émanant des établissements d'enseignement et de recherche français ou étrangers, des laboratoires publics ou privés. 


\title{
Experimental Thermomechanic study of newtonian and non newtonian suspension flows
}

\author{
M. Gradeck ${ }^{2 *}$, B. F. Z. Fagla ${ }^{1}$, C. Baravian ${ }^{2}$ and M. Lebouché ${ }^{2}$ \\ ${ }^{1}$ LERTI - Ecole Polytechnique d'Abomey Calavi - 01 BP 2009 Cotonou - BENIN \\ ${ }^{2}$ LEMTA CNRS UMR 7563 - 2, avenue de la forêt de haye BP 160 - 54504 Vandoeuvre lès Nancy Cedex FRANCE \\ * corresponding author : michel.gradeck@ensem.inpl-nancy.fr
}

\begin{abstract}
:
Hydrodynamic and thermal analysis of suspension flows is a difficult but fundamental task for the sterilization problems of particle charged fluids in food industries and also in any process where we can find flow with seeded particles. Nowadays, the influence of solid suspended particles on the flow is not yet a well known problem. Nevertheless, the solid particles have a strong influence on the rheological mixture's behaviour. The new approach of these solid-liquid suspension flows that we propose is based on experimental data and theoretical considerations. We use hard sphere approach in order to model the pressure drop assuming the mixture as an effective continuous medium. On these bases, we present a model of the heat transfer at the wall.
\end{abstract}

Key words :

suspension, solid-liquid, rheology, heat transfer

\section{Nomenclature}

\section{Latin symbols}

$\begin{array}{ll}C p & \text { specific heat }(\mathrm{J} /(\mathrm{kg} \cdot \mathrm{K}) \\ D & \text { diameter of the test section }(\mathrm{m}) \\ h(z) & \text { local heat transfer coefficient }\left(\mathrm{W} \cdot \mathrm{m}^{-2} \cdot \mathrm{s}^{-1}\right) \\ k & \text { thermal conductivity }\left(\mathrm{W} \cdot \mathrm{m}^{-1} \cdot \mathrm{K}^{-1}\right) \\ L & \text { total length of the test section }(\mathrm{m}) \\ L_{T} & \text { length of thermal establishment } \\ \dot{m} & \text { mass flow rate }\left(\mathrm{m}^{3} \cdot \mathrm{s}^{-1}\right) \\ T_{e} & \text { entry temperature }(\mathrm{K}) \\ T_{m}(z) & \text { mean temperature }(\mathrm{K}) \\ T_{w}(z) & \text { local wall temperature }(\mathrm{K}) \\ U_{d} & \text { mean velocity }\left(\mathrm{m} \cdot \mathrm{s}^{-1}\right) \\ z & \text { abscissa }\end{array}$




\section{dimensionless numbers}

Cf friction factor

$G z \quad$ mean Graetz number, $G z=\dot{m} \cdot C_{p} / k \cdot L$

$N u(z)$ local Nusselt number, $N u(z)=h(z) . D / k$

$\overline{N u} \quad$ mean Nusselt number, $\overline{N u}=\frac{1}{L-L_{T}} \int_{L_{T}}^{L} N u(z) d z$

Pr Prandtl number of the fluid, $\operatorname{Pr}=\mu . C p / k$

$\operatorname{Pr}_{\text {eff. }} \quad$ effective Prandtl number of the suspension, $\operatorname{Pr}_{\text {eff. }}=\mu_{\text {eff. }} . C p / k$

Re Reynolds number, $\operatorname{Re}=\rho \cdot U_{d} . D / \mu$

$R e_{e f f}$ effective Reynolds number, $\operatorname{Re}_{e f f .}=\rho \cdot U_{d} . D / \mu_{\text {eff. }}$

$\operatorname{Re}_{\text {sing. }}$ Reynolds number based on the viscosity of the suspending fluid, $\operatorname{Re}_{\text {sin } g .}=\rho \cdot U_{d} . D / \mu_{0}$

$X^{+} \quad$ Cameron number, $X^{+}=2 . z / D \cdot \operatorname{Re} \cdot \operatorname{Pr}=2 . z / D \cdot P e$

\section{greek symbols}

$\Delta P \quad$ pressure drop $(\mathrm{Pa})$

$\Phi \quad$ volume suspension fraction

$\Phi_{p} \quad$ random packing fraction $\left(\phi_{p} \approx 0,637\right)$

$\varphi_{w} \quad$ wall heat flux $\left(\mathrm{W} . \mathrm{m}^{-2}\right)$

$\mu \quad$ dynamic viscosity (Pa.s)

$\mu_{0} \quad$ dynamic viscosity of the liquid (Pa.s)

$\mu_{e f f} \quad$ dynamic viscosity of the suspension (Pa.s)

$\mu_{r} \quad$ relative viscosity

$\rho \quad$ mass volume $\left(\mathrm{kg} \cdot \mathrm{m}^{-3}\right)$ 


\section{Introduction}

Most of the fluids or mixtures that are produced by the food industries have a complex rheological behaviour. The knowledge of all their properties is essential to model different stages of the process. The product is pumped, heated, cooled and finally conditioned . During the process, it undergoes several physical and chemical transformations while it will be transformed. Hydrodynamic and thermal analysis of Newtonian and non Newtonian suspension flows is a fundamental task for the sterilization of fluids charged with solid. Nowadays, the solid particles influence on the flow is not yet well known. Nevertheless, this influence determines the mixture's rheology, that is to say all the thermal and mechanical problems encountered during the process. We can easily find a lot of works on solid-liquid flow in the literature ; the usual approach of this complex problem consists in taking account of all of the phenomena (particle-particle interaction, particle-liquid interaction, wall-liquid interaction and wall-particle interaction) in order to be able to solve the transport and energy equations. But this is still an open problem due to its complexity.

Several studies on flow and heat transfer have been conducted in the past. Durand [1] and Kyokay [2] defined four main patterns for the horizontal flow of suspension depending on the shape and size of particles, velocity of the mixture, volume concentration of the solid phase, diameter of the pipe, viscosity of the liquid, specific weight of the liquid and solid. These regimes are the so called symmetric, asymmetric, moving bed and stationary bed and they essentially depend on the sedimentation velocity of the solid phase. For each pattern, a law can be defined for the prediction of pressure drop. It seems to be very important to know the pattern because the velocity field is greatly influenced by it and thus the dynamic and thermal boundary layers. That is the reason why several authors were interested in studying the relations between the radial concentration, the velocity field and the heat transfer at the wall. Ayukawa [3] gave a model for the turbulent velocity field of a heterogeneous mixture of solid particles in water flowing between two parallel planes. The velocity field is given as a 
function of the radial solid concentration. Furuta et al. [4] reported that the radial concentration is a function of the size, density of particles and liquid velocity as well. Mills et al. [5] proposed a model based on lubrication forces. Their model clearly shows that there is, as expected experimentally, a peak of concentration in the middle of the flow. However the solid concentration is lower in the immediate vicinity of the wall and the motion of these particles will greatly disturb the thermal boundary layer. In a recent study, Hestroni and al. [6] experimentally showed the effect of particle motion and rotation on the temperature distribution and on the heat transfer.

Sure, all these studies are very interesting because they propose models based on physical considerations. The aim of present study is not to propose a flow model similar to previous studies. Some models can be applied to predict friction laws and heat transfer. The flow of Newtonian or non Newtonian liquids charged with equal density hard spheres is studied here. The dimensions of the particles are not negligible compared with those of the tube $(0,1$ to 0,2 typically). In the first part of the study, we show that the pressure drop can be modelled using a simple expression of the effective viscosity of the mixture given by Quemada [7]. In the second part, the experimental local heat transfer coefficients are compared to the classical laws ; all these experiments allow us to propose a physical explanation of the heat transfer for charged flows.

\section{Experimental set-up}

\subsection{Flow loop and test section}

The schema of the loop used in these experiments is shown on Fig. 1. The mixture (solidliquid) is supplied from a tank and circulates thanks to a centrifugal pump. After the mixture has passed through the test section, it is collected downstream in the main tank. The temperature of the solid-liquid suspension is kept constant at the entrance of the test section thanks to an upstream heat exchanger. The role of the upstream secondary tank is to dissipate 
all the pulsations generated by the centrifugal pump. The mixture flow rate is measured thanks to an electromagnetic flowmeter.

The test section was located at a distance of $2.3 \mathrm{~m}$ from the exit of the secondary tank in order to ensure that the flow was established at its entrance. The test section consists of a heated copper pipe, diameter $0.03 \mathrm{~m}$, length $2.15 \mathrm{~m}$, instrumented with 58 thermocouples inserted flushed to the wall. These thermocouples allow measurements of the wall temperature as a function of the axial distance from the entrance of the test section. The heating of the copper pipe is ensured by a set of electric resistance which are coiled around the pipe. These electric resistances can supply a maximum wall heat flux of about $15000 \mathrm{~W} / \mathrm{m}^{2}$ (see Fig. 2). The data acquisition system is a AOIP system (SAM 70). A differential pressure transducer measures the global pressure drop. In Table 1, we give the exact location of each thermocouple.

\subsection{Mixture}

The Newtonian liquid phase we used was an aqueous solution of glucose at different concentrations. The viscosity of these solutions varied from 0.001 Pa.s (water viscosity) to 0,06 Pa.s. The non Newtonian liquid is an aqueous carboxymethylcellulose solution (CMC). The rheology of suspended fluids has been quantified using a stress controlled rheometer adapted with a plate and cone geometry (diameter $=6 \mathrm{~cm}$; aperture angle $\left.=2,1^{\circ}\right)$. Rheograms of CMC are modelled with the following law :

$$
\mu=\frac{\mu_{0}}{1+\sqrt{0,032 \cdot \dot{\gamma}}}
$$

where $\mu_{0}=0,315$ is the viscosity of $\mathrm{CMC}\left(20^{\circ} \mathrm{C}\right)$ at zero shear stress.

The Reynolds number is calculated using this viscosity $(\mu)$. The generalized Reynolds number of Metzner - Reed is not used in this study. 
The solid phase is constituted by alginate hard spheres. These spheres are obtained by the fall (drop by drop) of an aqueous solution of alginate in another aqueous solution of $\mathrm{CaCl}_{2}$; this last solution is used in order to ensure the polymerisation of the alginate drop.

In Table 2, we summarize the essential physical properties of each phase.

\section{Experimental results}

\subsection{Rheology - Pressure drop}

In the case of charged suspensions, the measure of the rheological properties can be a problem using a classical rheometer. In our case, the solid particles have a mean diameter of about 4.4 $\mathrm{mm}$ so we can not measure the mixture's properties using a stress-controlled rheometer.

The other method to measure these properties is to measure the global pressure drop ; the rheological properties of the mixture will be determined assuming some hypotheses. If we make the assumption that our mixture behaves like a newtonian equivalent fluid for example, it is easy to refer to an equivalent viscosity for each particle concentration. But this method is questionable because there is no sufficient reason to act that the solid-liquid mixture is an equivalent Newtonian fluid: the radial solid concentration is not constant so the viscosity of the mixture will evolve on a radius. Newitt et al. [8] gave a simple expression of the pressure drop in the case of a moving bed. For other regimes like symmetric or asymmetric ones, most authors considered the mixture as an equivalent fluid (newtonian or not). The works of Cheng [9] are concerned with non newtonian fluids and these authors studied the flow of finest dispersion like paint. However the most common way to model pressure drop for solid-liquid flow is to express the two-phase pressure drop as a function of the single-phase one. That is the case of several works like those of Cheng [9] as well, Kemblowski et al. [10], Takahashi [11], Chhabra [12] and Hoareau [13]. 
If we take a look at the different rheological models suitable for suspension of hard spheres, we can mentioned of course the model of Einstein [14] which is valid for dilute suspensions $(\Phi<0.5 \%)$ but in our cases the solid concentration is always greater than this limit. Krieger [15] give available model for the effective mixture viscosity (liquid can be Newtonian or not) as a function of the liquid viscosity and the volume concentration of the solid phase :

$\mu_{e f f}=\mu_{0}\left(1-\frac{\phi}{\phi_{P}}\right)^{-q}$

where $\Phi_{P}$ is the packing fraction.

Quemada [7] takes the value of 2 for the q exponent. Let us precise that all these works have been validated for micrometric particles and not yet for millimetric particles although there is no theoretical considerations which prevent to transpose this law for millimetric particles. In figure 3, the viscosity of our mixture determined through pressure drop-flow rate relationship for homogeneous fluids is in very good agreement with Quemada [7] law. Validation of the Quemada model has been made using a glucose solution (Newtonian fluid with $\mu=0,06$ Pa.s). The Reynolds Number based on the viscosity of the carrier fluid is equal to 934.

The estimation of pressure drop for solid-liquid flow has been widely studied in the past. In this work, we consider our mixture as an effective fluid. In Fig. 4, we present the evolution of the solid-liquid friction coefficient as a function of the effective Reynolds number for several concentrations of particles (volume concentration). For laminar flow, the experimental points are supposed to fit the $16 / \mathrm{Re}$ curve. The Blasius law is the reference for turbulent flow $\left(0,316^{*} \mathrm{Re}^{-0,25}\right)$. These curves have been obtained in the case of Newtonian liquid (water and glucosic suspensions) and Non Newtonian ones (CMC). We can noticed that all the experimental points are located on master curves independent of suspension concentration, this means that the chosen parameters (Reynolds number constructed with the effective viscosity) are the appropriate ones to describe the pressure drop of such mixtures. In fact, the 
effective viscosity takes into account all the dissipations induced by the particles interacting each other, the particles interacting with the fluid,... So the dissipation measured by the way of the pressure drop on a given length of the pipe integrates all of these dissipations and not only the viscous dissipation at the wall. In the case of a Newtonian suspending phase, the laminar-turbulent transition is very smooth and the experimental points leave the theoretical laminar curve for a Reynolds number around 1000. This advanced transition is due to the particle-particle interaction inducing finite size perturbations. In the case of a pseudoplastic liquid, this transition is classically retarded because of higher shear rates near walls due to its shear-thinning behavior.

Our approach allows a significant simplification of the classical laws for mixtures: one only needs to know the (eventually non-newtonian) viscosity of the liquid phase, the exact value of the solid phase concentration and the value of the packing fraction. To describe the pressure drop of any solid-liquid mixture, there is no need of others additional parameters such as the ratio between the characteristic dimension of the solid phase and the pipe diameter, shape of the solid phase,...

\subsection{Heat transfer}

\subsubsection{Preliminary calculations}

The local heat transfer coefficient was calculated as :

$h(z)=\frac{\varphi_{w}}{T_{p}(z)-T_{m}(z)}$

where $\varphi_{w}$ is the wall heat flux density. It is supposed to be constant over all the pipe length ; the axial conduction in the $\mathrm{z}$ direction is assumed to be zero. $T_{w}(z)$ is the local surface temperature at the $\mathrm{z}$ abscissa; $T_{m}(\mathrm{z})$ is the mean temperature of the fluid at the $\mathrm{z}$ abscissa. This temperature is obtained by a balance between the total heat flux transferred from the entrance of the pipe to the $\mathrm{z}$ abscissa : 


$$
T_{m}(z)=T_{e}+\frac{\pi \cdot D \cdot \varphi_{w} \cdot z}{\dot{m} \cdot C_{p}}
$$

So, the local Nusselt number can be defined as:

$$
N u(z)=\frac{h(z) \cdot D}{k}=\frac{\varphi_{w} \cdot D}{k \cdot\left(T_{p}(z)-T_{m}(z)\right)}
$$

The thermal conductivity is estimated at the mean temperature of the fluid, $T_{m}(z)$.

The entrance temperature $T_{e}$ is adjusted at $20^{\circ} \mathrm{C}$ with an uncertainty of about $0,1^{\circ} \mathrm{C}$. The surface averaged heat flux density is calculated from the measured electric power supplied by the coiled resistance. Finally, a standard uncertainty analysis indicates that the uncertainty in the heat transfer coefficient was of the order of $10 \%$.

\subsubsection{Influence of the Reynolds number and heat flux density}

Experiments have been carried out for different Reynolds numbers and different heat density fluxes both for single-phase and solid-liquid flows. Fig. 5 shows the results of experiments in the case of single-phase flow; for laminar flow, there is a very good agreement between the experimental results and the correlation established by Mizushina [16] and modified by Moudachirou [17]:

$$
N u(z)=1,64 \cdot\left[-\left(\frac{d \mu}{\mu d T}\right) \frac{\varphi_{w} \cdot D}{2 \cdot k}\right]^{0,14} \cdot\left(X^{+}\right)^{-1 / 3}
$$

In the case of higher Reynolds numbers $(\mathrm{Re}=2787)$, the Nusselt number shows a particular evolution along the pipe. It is decreasing in the entrance part of the pipe as predicted by Eq. 6 and then remains constant until the exit. This is typical of a flow transition between laminar to turbulent regimes. While the thermal boundary layer is confined in the viscous sublayer, it can grow normally, but when its thickness reached the turbulent core, its growth is brutally stopped by the turbulent diffusivity. That is why the Nusselt number remains constant. The previous correlation (Eq. 6) is not valid anymore; the only one that can be used now is the one established for turbulent flows by Chilton and Colburn [18] : 
$\overline{N u}=0,023 \cdot \operatorname{Re}^{0,8} \cdot \operatorname{Pr}^{1 / 3}$

The thermal dependence of the fluid is correctly taken into account by the Mizushina's correction of Eq. 6 (Figure 6).

\subsubsection{Influence of the volume fraction}

The influence of the particle volume fraction is clearly highlighted in figure 7 . If we compare the evolution of the local Nusselt number for single-phase case and two-phase case (3\%) for the same Reynolds $(\operatorname{Re}=1499)$, we can notice that not only the Nusselt number increases but it remains constant for a Cameron number of about $10^{-4}$ (Fig. 7a) : the transfer is like a turbulent one. When the Reynolds number increases (Fig. 7b), the turbulent transfer arises at a distance from the entrance of the pipe as much shorter as the Reynolds number is high. The local Nusselt number increases as the volume fraction is increased (Fig. 8). But we can noticed that the Nusselt number evolution is the same that in the case of a single phase turbulent flow, even when the flow is laminar. Naturally, when the volume fraction is increased, the Nusselt number is increased as well (Fig. 9) because the turbulence effects are as much greater as the volume fraction is high. As the mixture rate is measured thanks to an electromagnetic flowmeter, Ud (the velocity of the mixture) is closed to the velocity of the liquid for weak values of the mass concentration.

\subsubsection{Mean Nusselt number}

The evolution of the mean Nusselt number can be modelled by taking into account in relation (6) the Reynolds number based on the effective viscosity (Fig. 10a) or based on the viscosity of the suspending fluid (Fig. 10b). In the case of a laminar flow, the Kays's [19] relation can be used:

$$
\overline{N u}=1,41 \cdot G z^{1 / 3}
$$

In the figure $10 \mathrm{a}$, the evolution of the mean Nusselt number is reported versus the effective Reynolds number. As we can see, the Nusselt number is still a function of both the mass 
particles concentration and the Reynolds number. When the Nusselt number is reported as a function of the single-phase Reynolds number, all the results seem to be only dependant on this parameter.

Regarding the expansion of the thermal boundary layer can explain the process. In the laminar case, the thermal boundary layer can grow and when its thickness reached the centre of the pipe the thermal regime is considered as established, so the Nusselt number will remain constant. In the case of a turbulent flow, the thermal boundary layer can grow while it is confined in the viscous sub-layer, but when its thickness reach the turbulent core, the turbulent diffusivity stops brutally this growth. The case of solid-liquid flow is very similar to the turbulent one. Near the wall, the local particle volume fraction is so low that we can consider that there is an annular single-phase ring where there is no particles; so, the thermal boundary layer can grow normally until it is stopped by the particle's core where the diffusivity due to the interactions between particles and the liquid phase plays a similar rule as the turbulent diffusivity. These mechanisms have been schematically summarized in Fig. 11.

\section{Concluding remarks}

In the present paper, a series of experiments of forced convection heat transfer in a horizontal circular pipe for solid-liquid mixtures were conducted. The analysis of the pressure drop leads us to a significant simplification of the approach of heterogeneous suspensions. The use of the effective viscosity in order to calculate the Reynolds number can be used for pressure drop predictions. Furthermore, in the case of heating, the classical laws established in the case of single phase flows are still valid in the case of solid-liquid flows; the experiments show that there is a good agreement while the flow is laminar and the thermal regime is not established. The Chilton-Colburn relation is valid for solid-liquid flows ; the Reynolds number must be calculated using the viscosity of the suspending phase alone. 


\section{References :}

[1] Durand, R., D. 1953. Minnesota Int. Hydraulics Conv., Proc. Int. Ass. for Hydraulics Research, 89.

[2] Kyokay O., 1981, Trajectory and diffusion of particles in solid liquid flow slurry pipeline, J. of Pipelines, 211-223.

[3] Ayukawa K., 1970. Velocity distribution and pressure drop of heterogeneously suspended flow in hydraulic transport through a horizontal pipe, First International Conference on the hydraulic transport of solid in pipes.

[4] Furuta T, Tsujimoto S., Okazaki M. and Toei R., 1978, Concentration distribution of particles in solid-liquid two-phase flow through vertical pipe, Kagaku Kogaku Ronbushu (4) 105 .

[5] Mills P. and Snabre P., 1995, Rheology and structure of concentrated suspension of hard spheres. Shear induced particle migration, J. Phys. (10) 1597-1608

[6] Hestroni G., Gurevitch M., Rozenblit R., Yarin L.P., Ziskind G., 2001, Effect of particle motion on the wall's thermal structure and on heat transfer, Int. J. Multiphase Flow (27) 393-413

[7] Quemada D., 1998, Rheological modelling of complex fluid I. The concept of effective volume fraction revisited, Eur. Phys. J. AP (1) 119-127.

[8] Newitt D. M., Richardson, J. F., Abbott, M. and Turtle R. B., 1955, Hydraulic conveying of solids in horizontal pipes, Trans. Instn Chem. Engrs (33) 93-113

[9] Cheng D. C. - H.. , 1970. A design procedure for pipeline flow of non-newtonian dispersed systems. Hydrotransport I, 1-4 September, paper J5.

[10] Kemblowski, Z... and Kolodziejski, J.; 1973, Flow resistance of non-newtonian fluids in transitional and turbulent flow. Int. Chem. Eng. (13) 265-279.

[11] Takahashi, I., 1978, Pressure drop of suspensions in heterogeneous flow. Hydrotransport V, 8-11 May, paper C5.

[12] Chhabra R.. P. , 1990. Motion of spheres in power law fluids at intermediate Reynolds numbers: an unified approach. Chem. Eng. Process, (28) 89-94.

[13] Hoareau, F., 1996, Etude dynamique et thermique de suspensions solides-liquides nonnewtoniennes en conduite. Ph. D. Thesis, UHP- Nancy I.

[14] Einstein, A., 1906, Ann. Phys., Lpz., 19, 289.

[15] Krieger, I. M., 1972, Rheology of monodisperse latices Advan. Colloid Interface Sci. 3, 111-136.

[16] Mizushina T., Ito R., Kuriwake Y., Yahikaza K., 1967. Boundary layer heat transfer in a circular tube to newtonian and non-newtonian fluids, Kagaku- kogaku (31) 250-255.

[17] Moudachirou I., 1992, transfert thermique et perte de charge pour un fluide de Herchel-Bulkley thermodépendant, PhD Thesis, UHP- Nancy I.

[18] Chilton T. H. and Colburn A. P., 1934, Ind. ENG. Chem.(26) 1183.

[19] Kays W.M., 1955, numerical solutions for laminar flow heat transfer in circular tubes, Trans. ASME (58) 1265. 


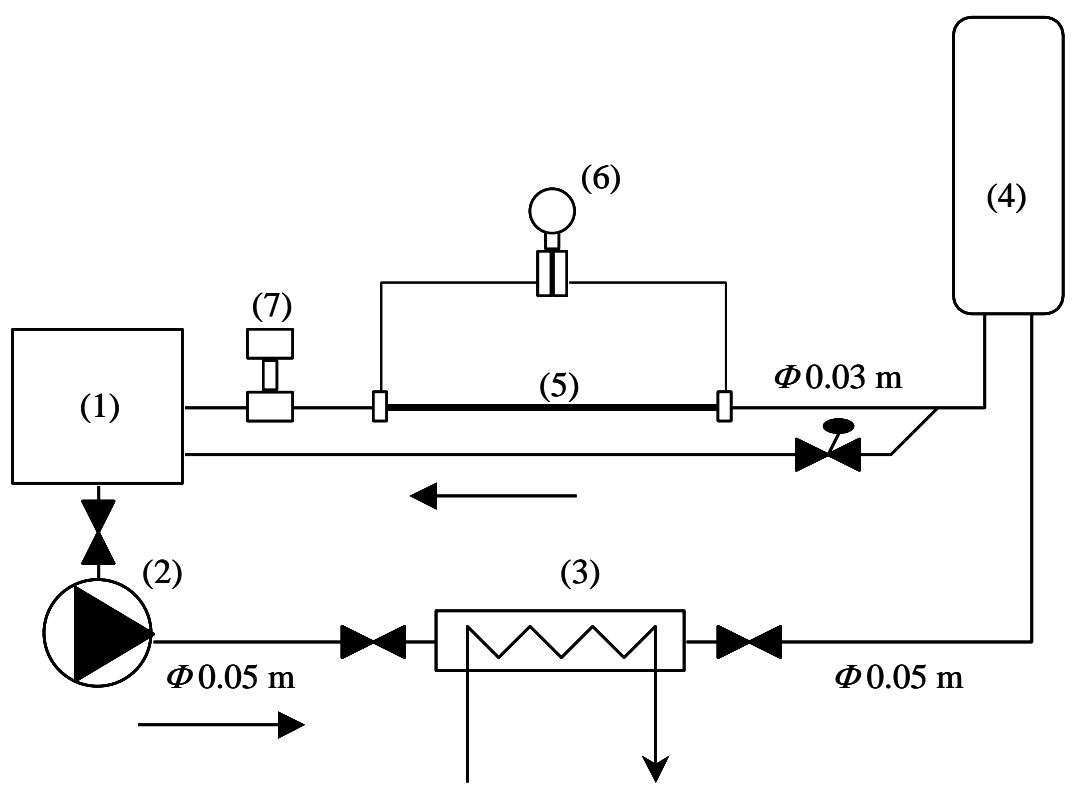

\begin{tabular}{|ll}
\hline (1) & Main tank \\
(2) & Centrifugal pump \\
(3) & Heat exchanger \\
(4) & Secondary tank \\
(5) & TEST SECTION \\
(6) & Differential pressure transducer \\
(7) & Magnetic flowmeter
\end{tabular}

Figure 1 : Schema of the flow loop 


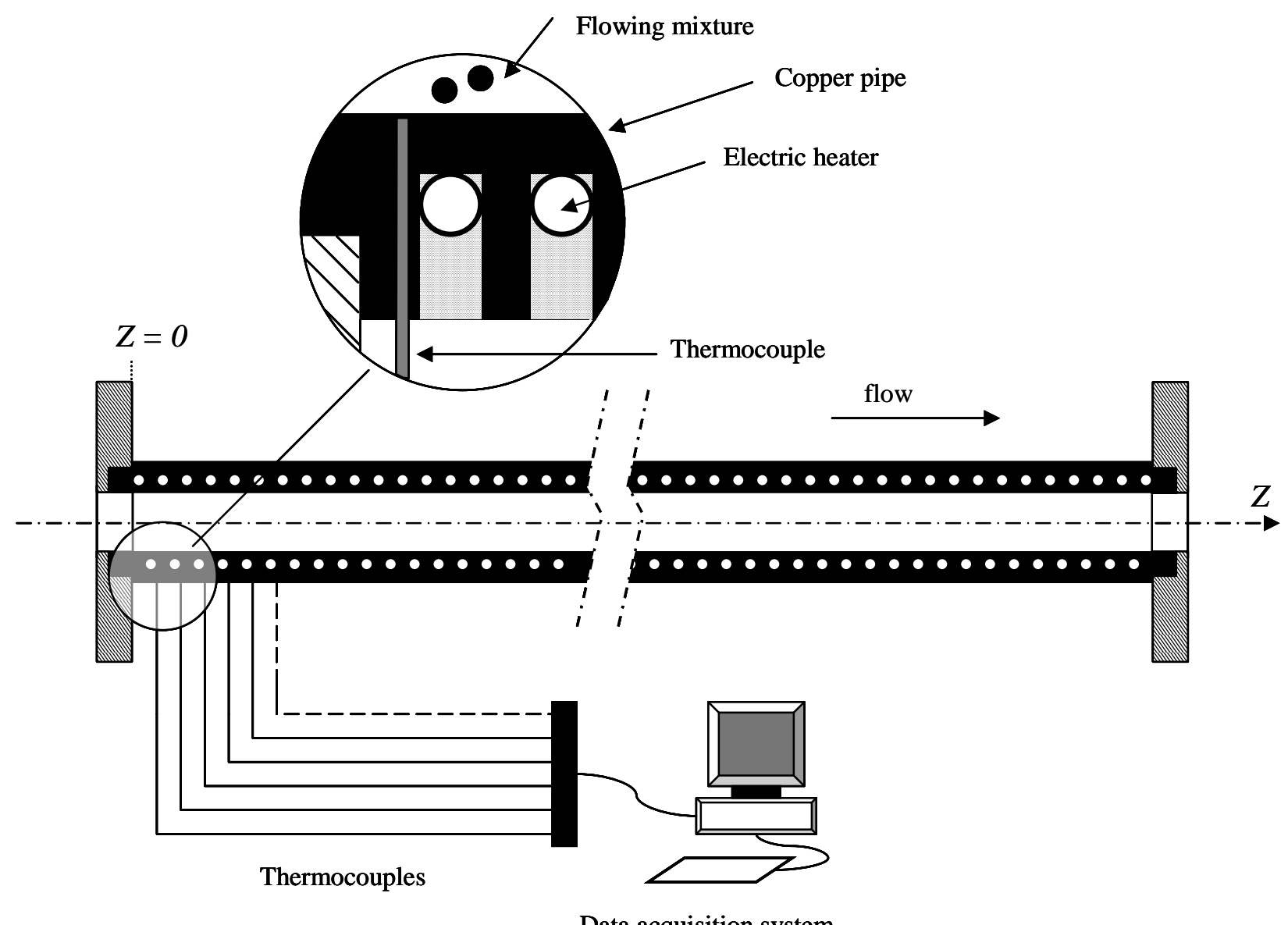

Data acquisition system

Figure 2: Details of the heated test section

\begin{tabular}{|l|c|c|c|c|c|c|c|c|c|c|c|c|}
\hline TC number & 1 & 2 & 3 & 4 & 5 & 6 & 7 & 8 & 11 & 12 & 13 & 14 \\
\hline $\mathrm{z}$ (mm) & 15 & 28 & 188 & 188 & 188 & 188 & 260 & 340 & 370 & 383 & 543 & 543 \\
\hline
\end{tabular}

\begin{tabular}{|l|c|c|c|c|c|c|c|c|c|c|c|c|}
\hline TC number & 15 & 16 & 17 & 18 & 21 & 22 & 23 & 24 & 25 & 26 & 27 & 28 \\
\hline $\mathrm{Z}$ (mm) & 543 & 543 & 615 & 695 & 725 & 738 & 898 & 898 & 898 & 898 & 970 & 1050 \\
\hline
\end{tabular}

\begin{tabular}{|l|c|c|c|c|c|c|c|c|c|c|c|c|}
\hline TC number & 31 & 33 & 34 & 35 & 36 & 37 & 38 & 41 & 42 & 43 & 44 & 45 \\
\hline $\mathrm{z}$ (mm) & 1080 & 1253 & 1253 & 1253 & 1253 & 1325 & 1405 & 1435 & 1448 & 1608 & 1608 & 1608 \\
\hline
\end{tabular}

\begin{tabular}{|l|c|c|c|c|c|c|c|c|c|c|}
\hline TC number & 46 & 47 & 48 & 51 & 52 & 53 & 54 & 56 & 57 & 58 \\
\hline $\mathrm{Z}(\mathrm{mm})$ & 1608 & 1680 & 1760 & 1790 & 1803 & 1963 & 1963 & 1963 & 2035 & 2115 \\
\hline
\end{tabular}

Table 1: Location of the TC 


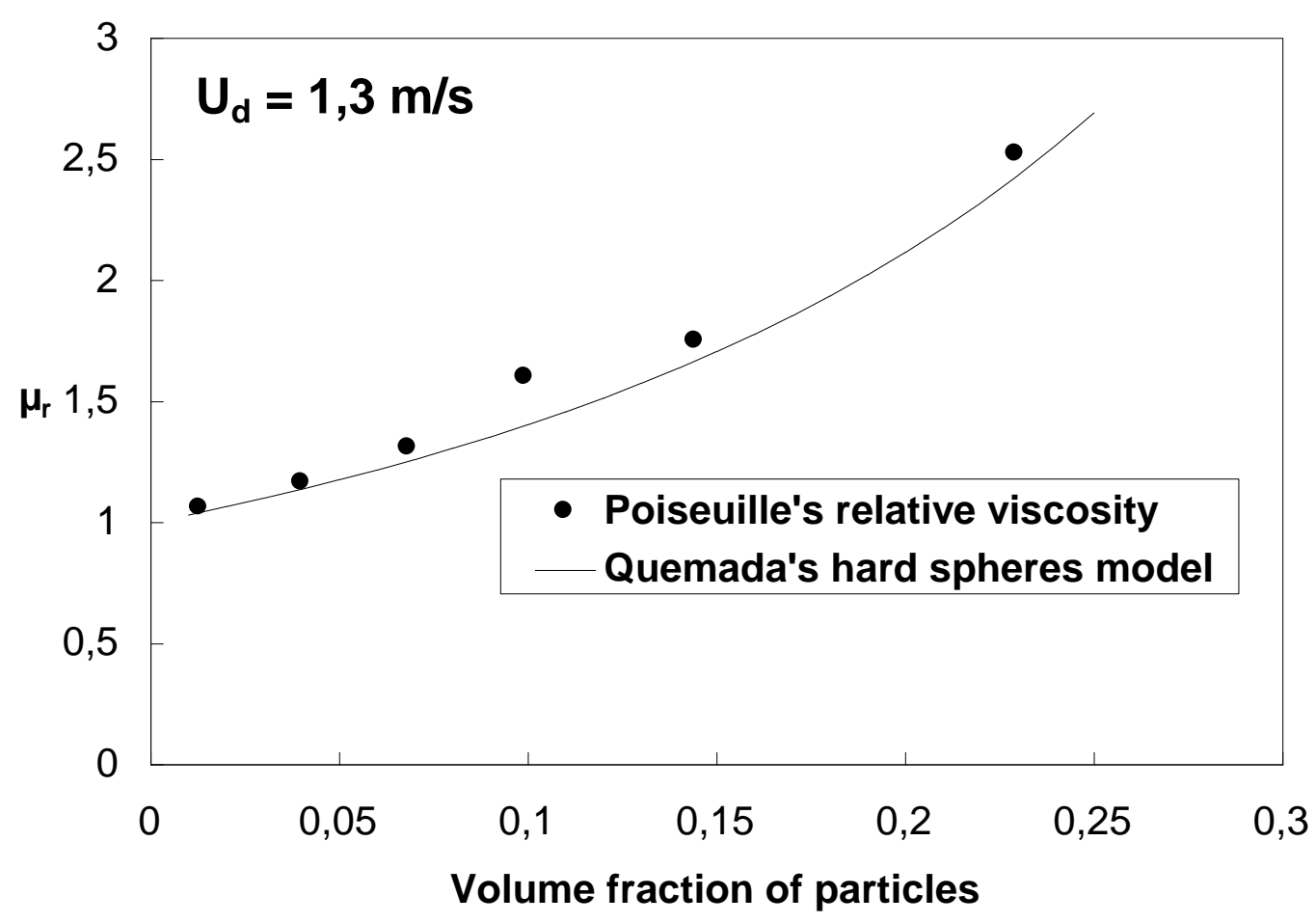

Figure 3 : evolution of the relative viscosity versus the solid concentration $-\mathrm{Re}_{\text {sing }}=934$ 


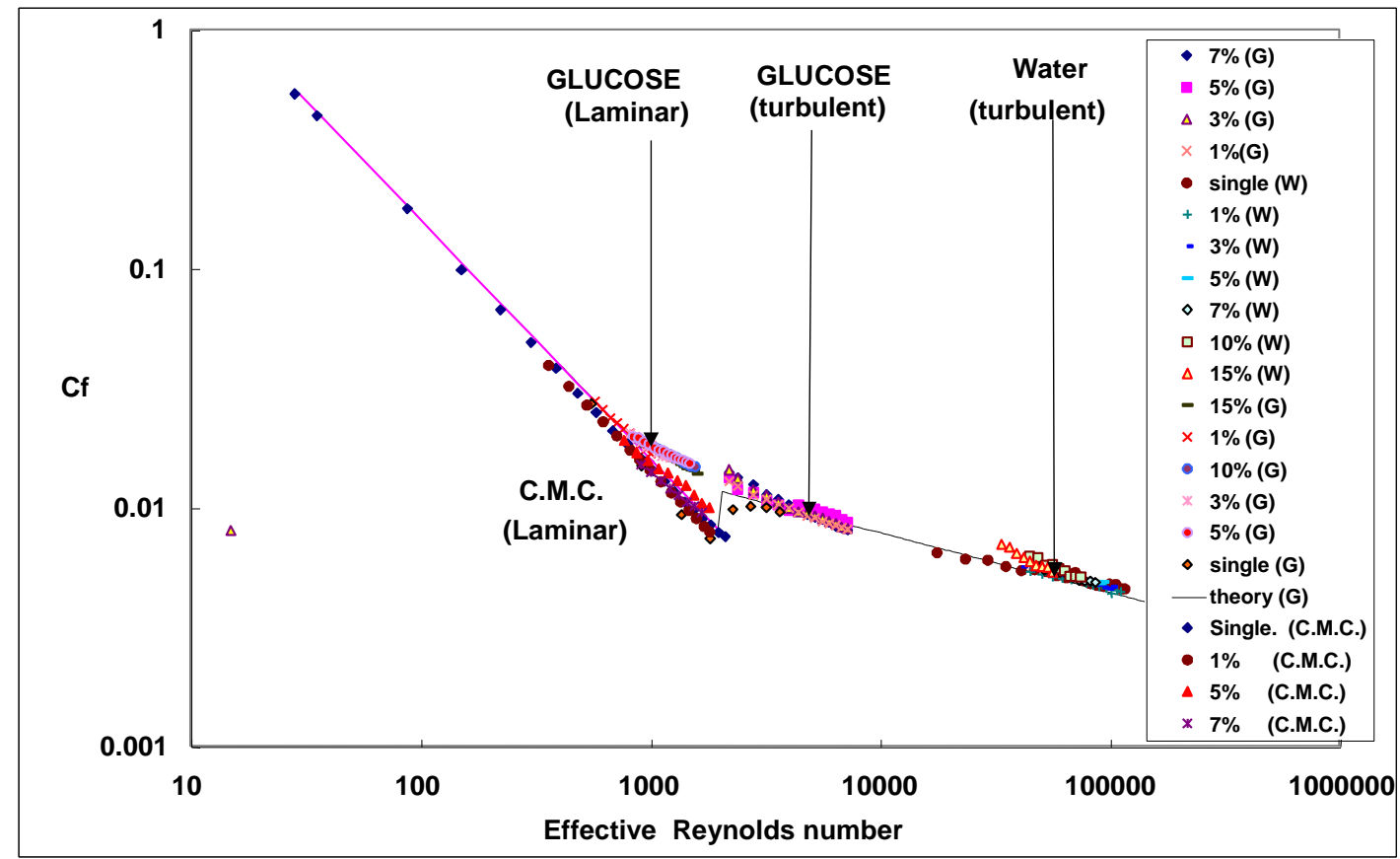

Figure 4 : friction factor versus the effective Reynolds number for different concentrations (\%) 


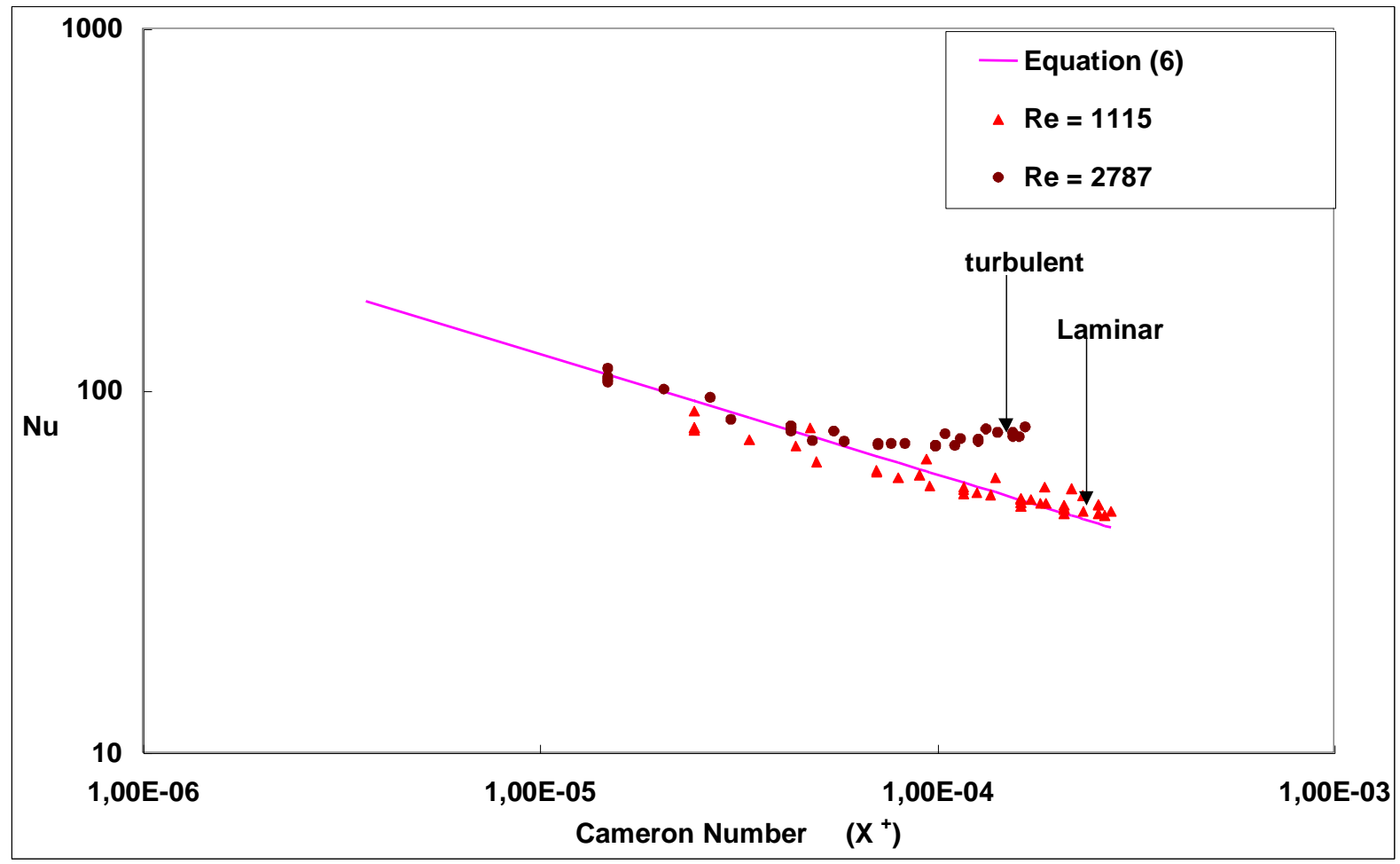

Figure 5: Evolution of the local Nusselt number according to the Cameron number _ case of single phase flow $\varphi_{w}=15.10^{3} \mathrm{~W} / \mathrm{m}^{2}$. 


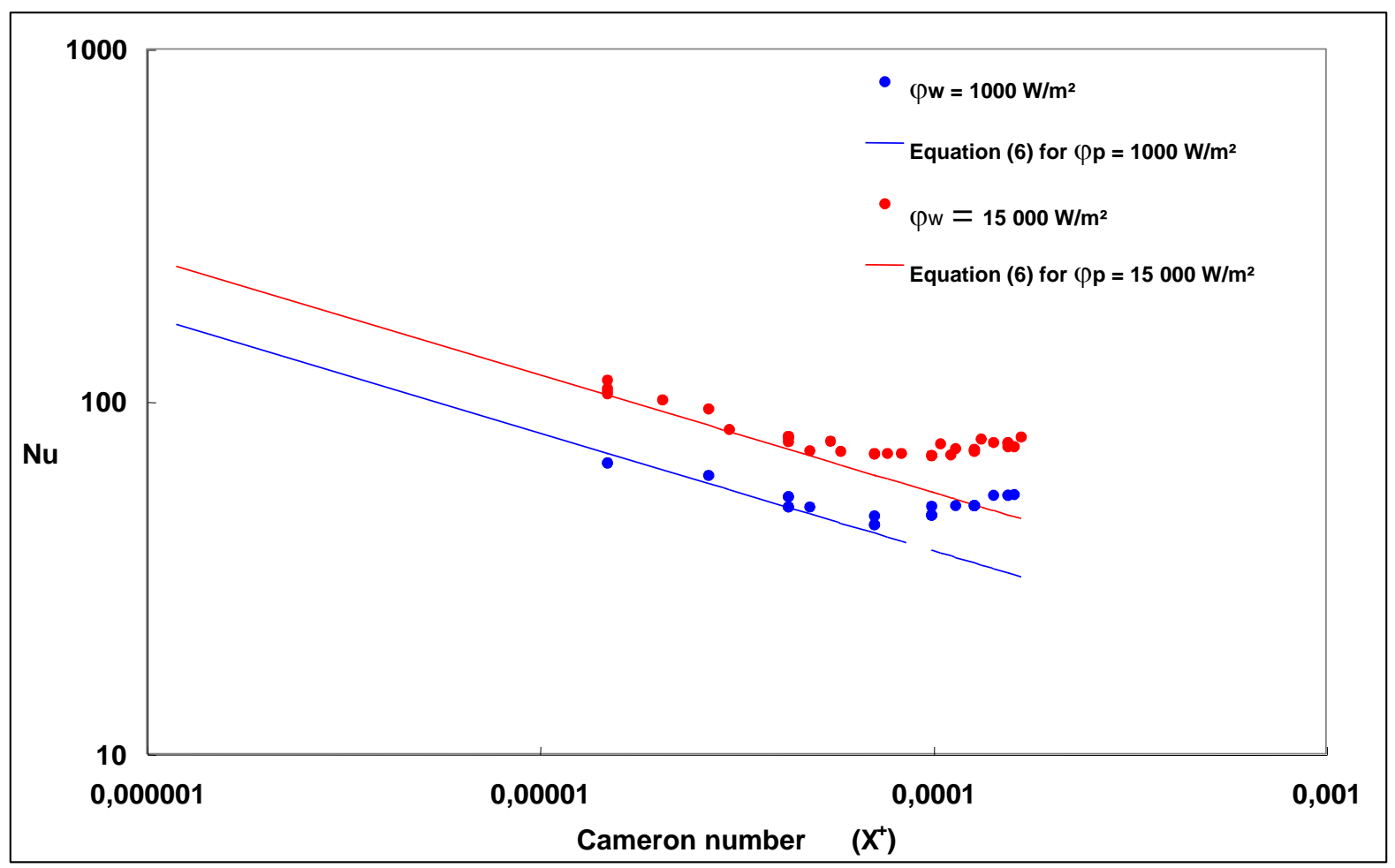

Figure 6: influence of the heat flux density (i.e the thermal dependancy of the fluid) In the case of a single phase flow _ $\operatorname{Re}_{\text {sing }}=2787$ 


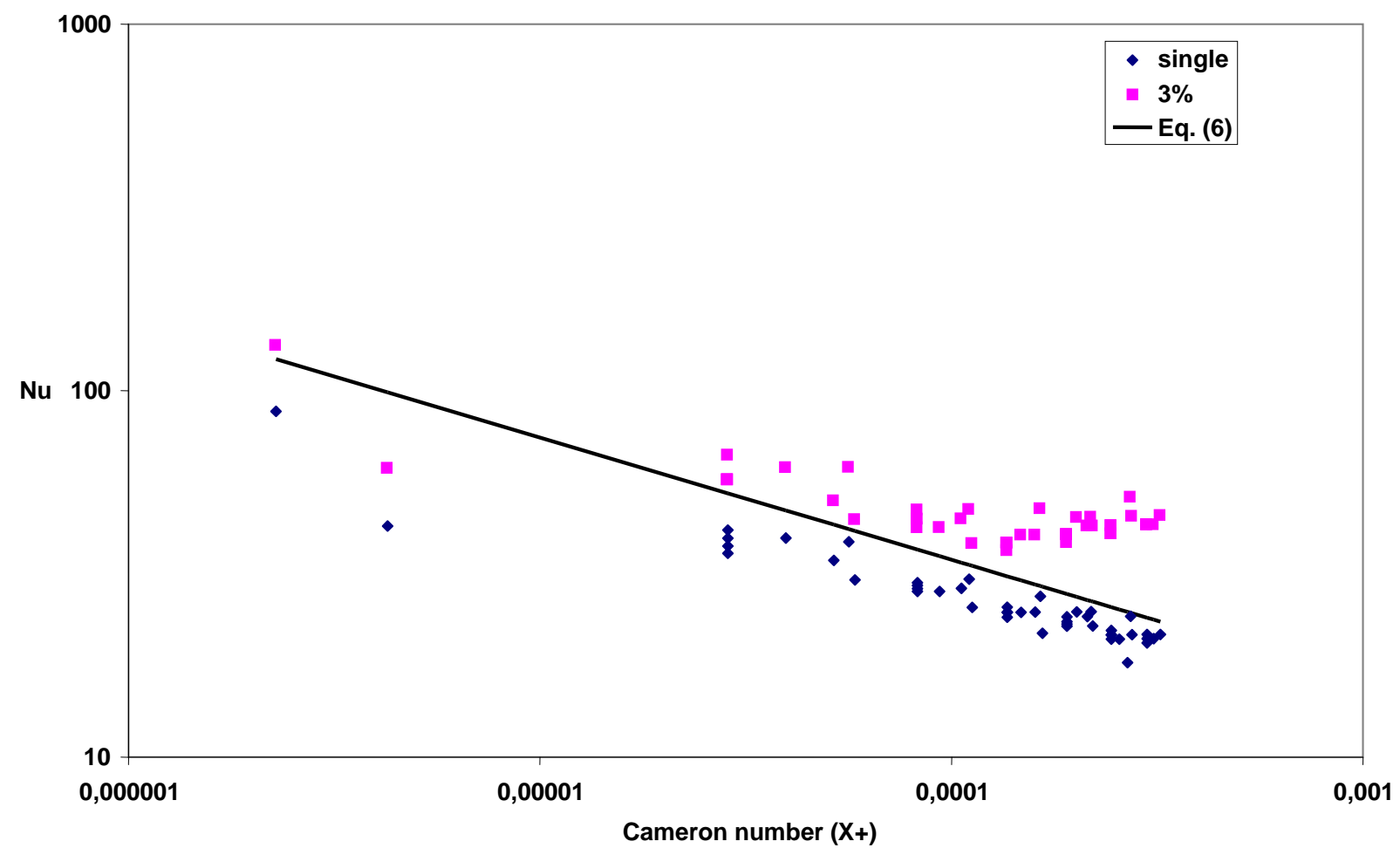

Figure 7a : Evolution of the local Nusselt number - comparison of the single phase case with the volume fraction $=3 \%-\operatorname{Re}_{\text {sing }}=1499$

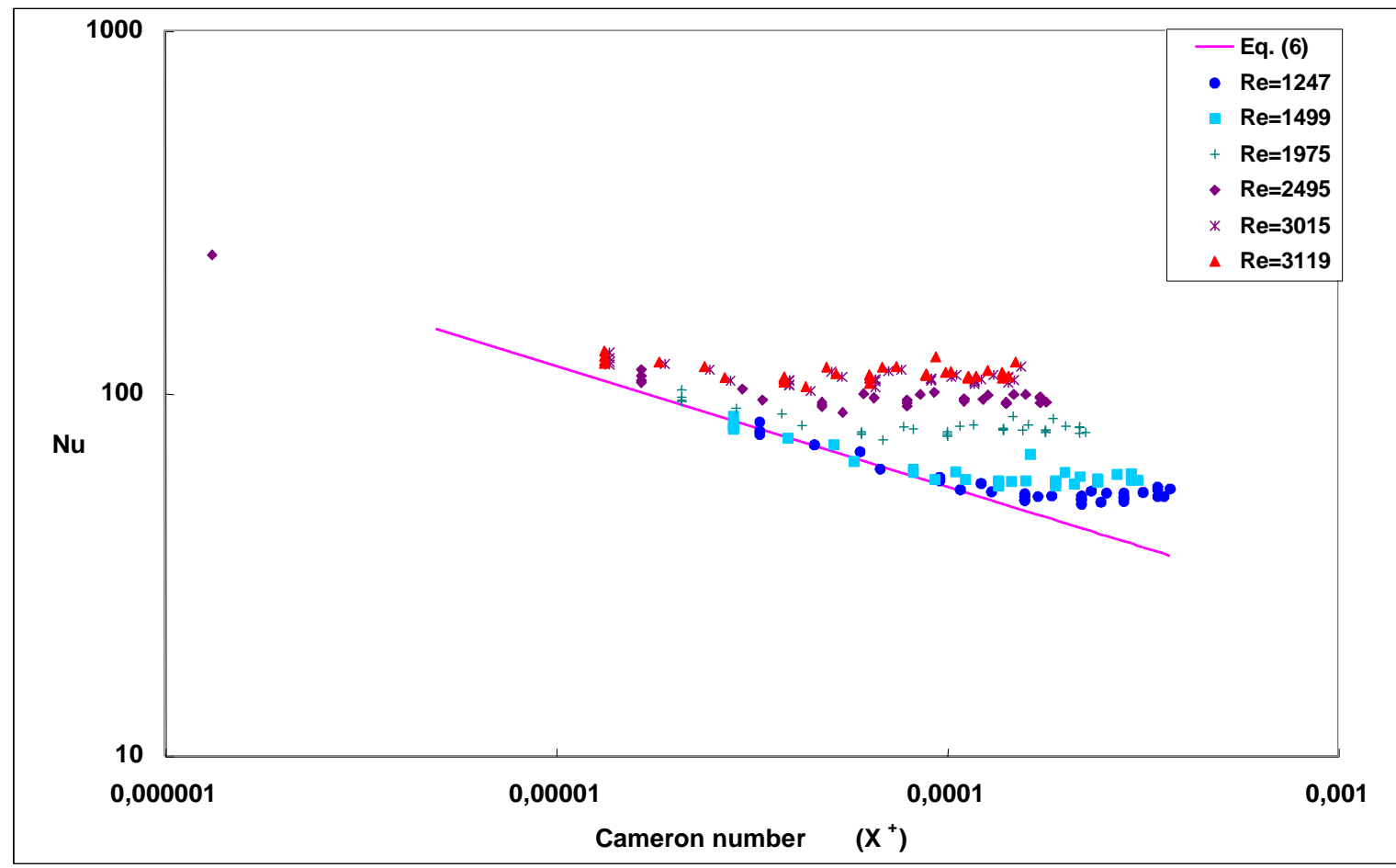

Figure 7b: Evolution of the local Nusselt number for different Reynolds numbers $\varphi_{\mathrm{w}}=15000 \mathrm{~W} / \mathrm{m}^{2}-$ volume fraction $=3 \%$ 


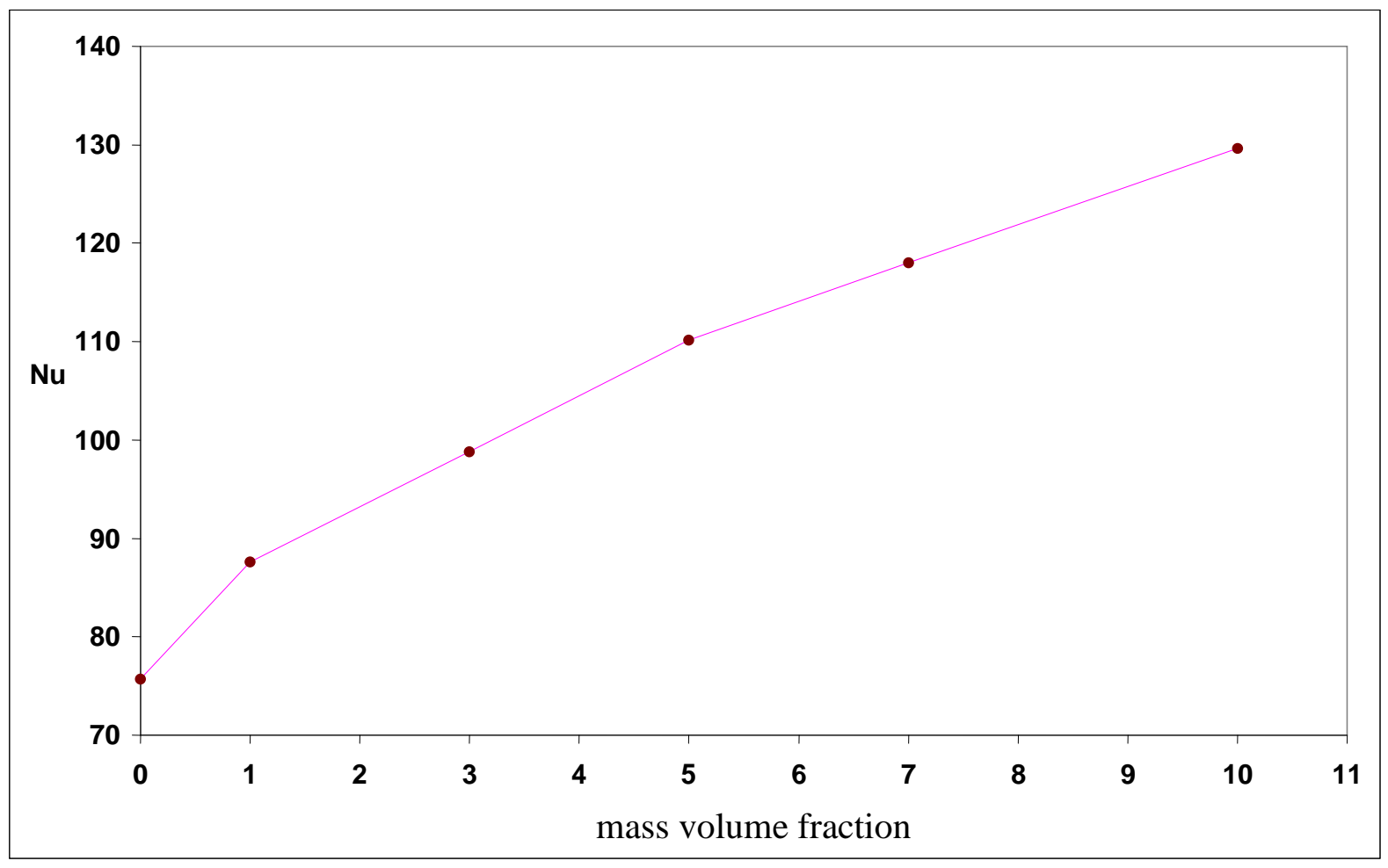

Figure 8 : Local Nusselt number $(X+\sim 10-4)$ versus mass volume fraction $\_\operatorname{Re}_{\text {sing }}=2787$ 


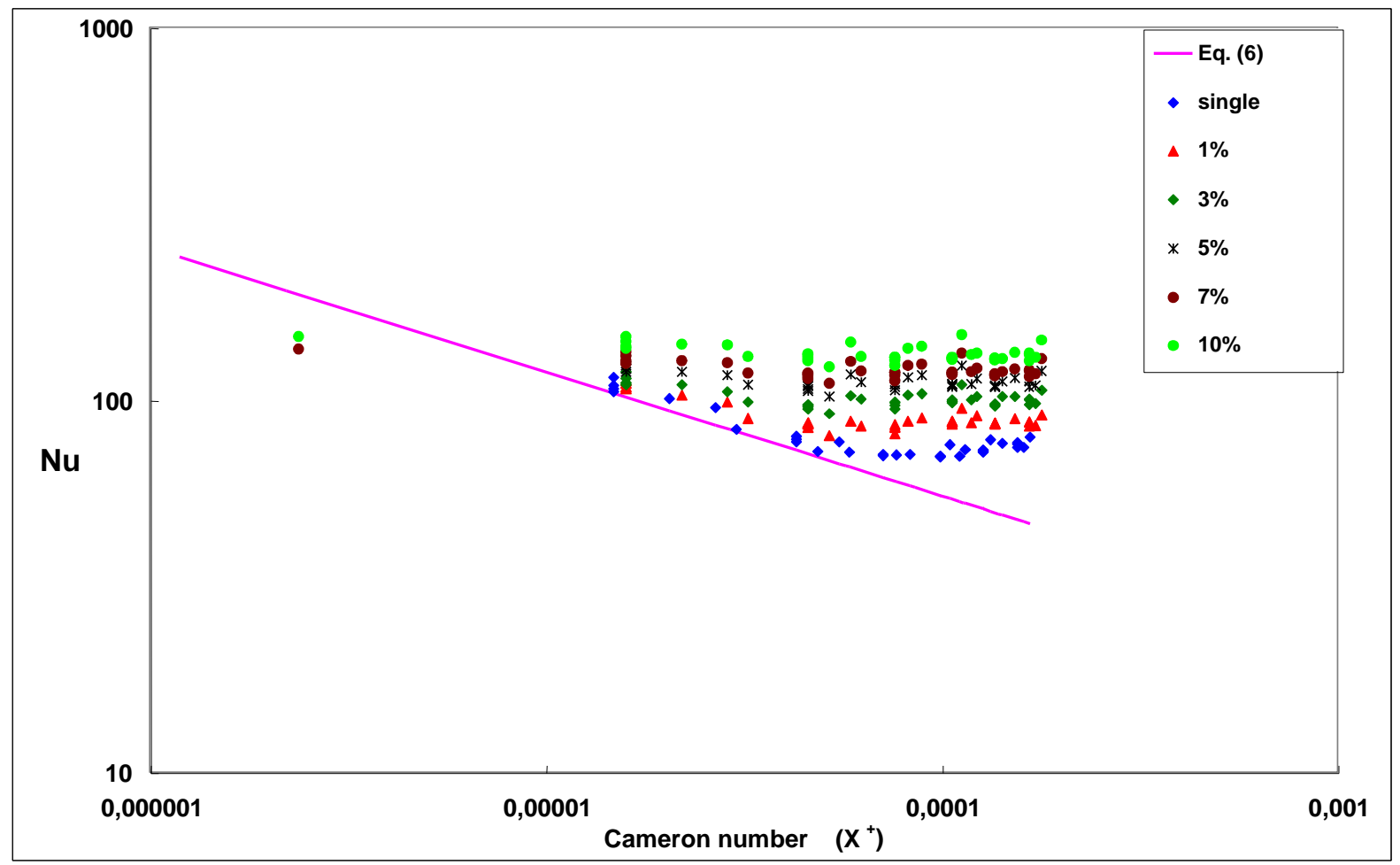

Figure 9: Evolution Nusselt number versus Cameron number $(\mathrm{X}+)$ for different volume fractions $-\varphi_{\mathrm{w}}=15000 \mathrm{~W} / \mathrm{m}^{2}$ and $\mathrm{Re}_{\text {sing }}=2787$ 


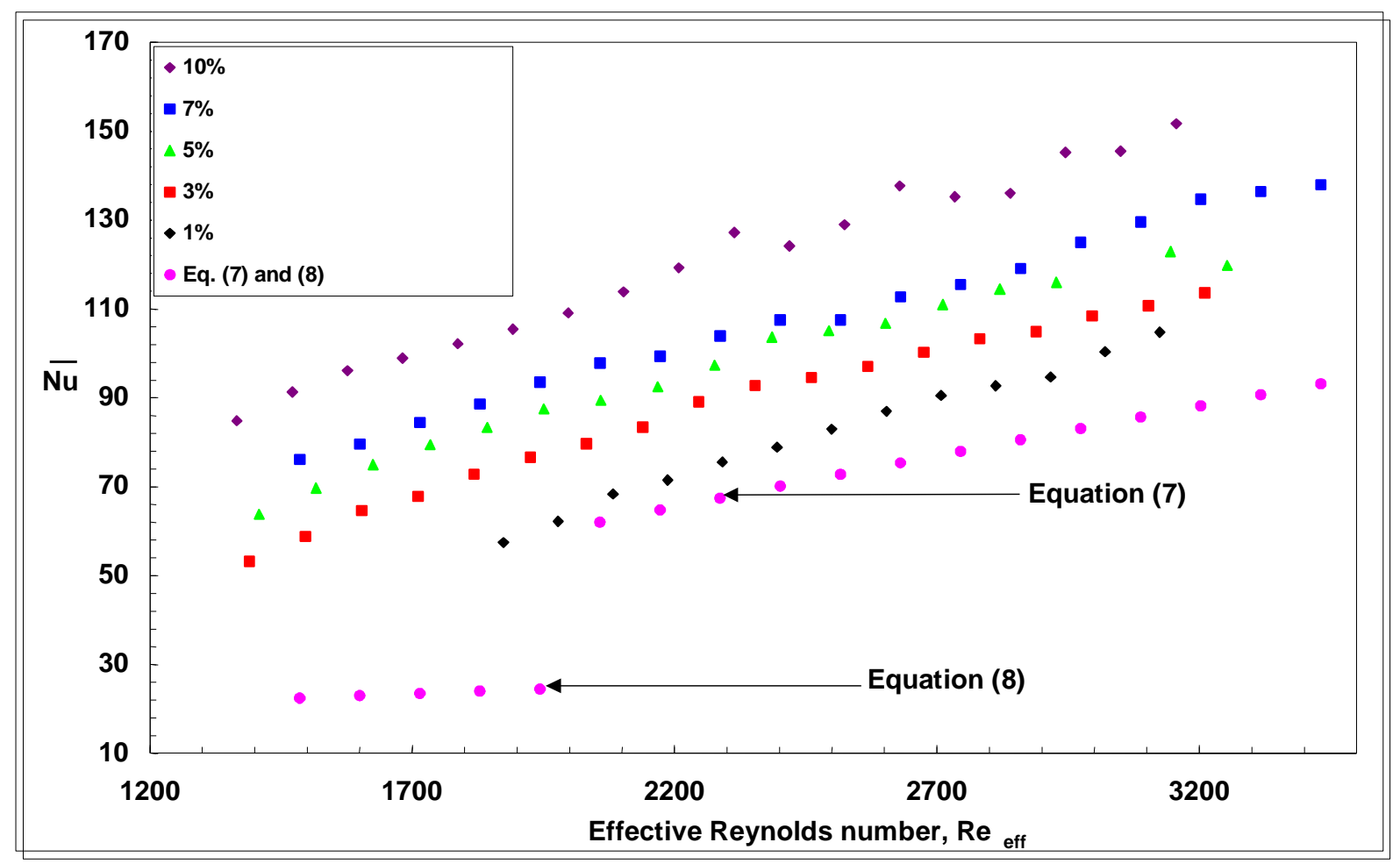

Figure 10a : Mean Nusselt number versus effective Reynolds number for several volume fractions $\varphi_{\mathrm{w}}=15.000 \mathrm{~W} / \mathrm{m}^{2}$.

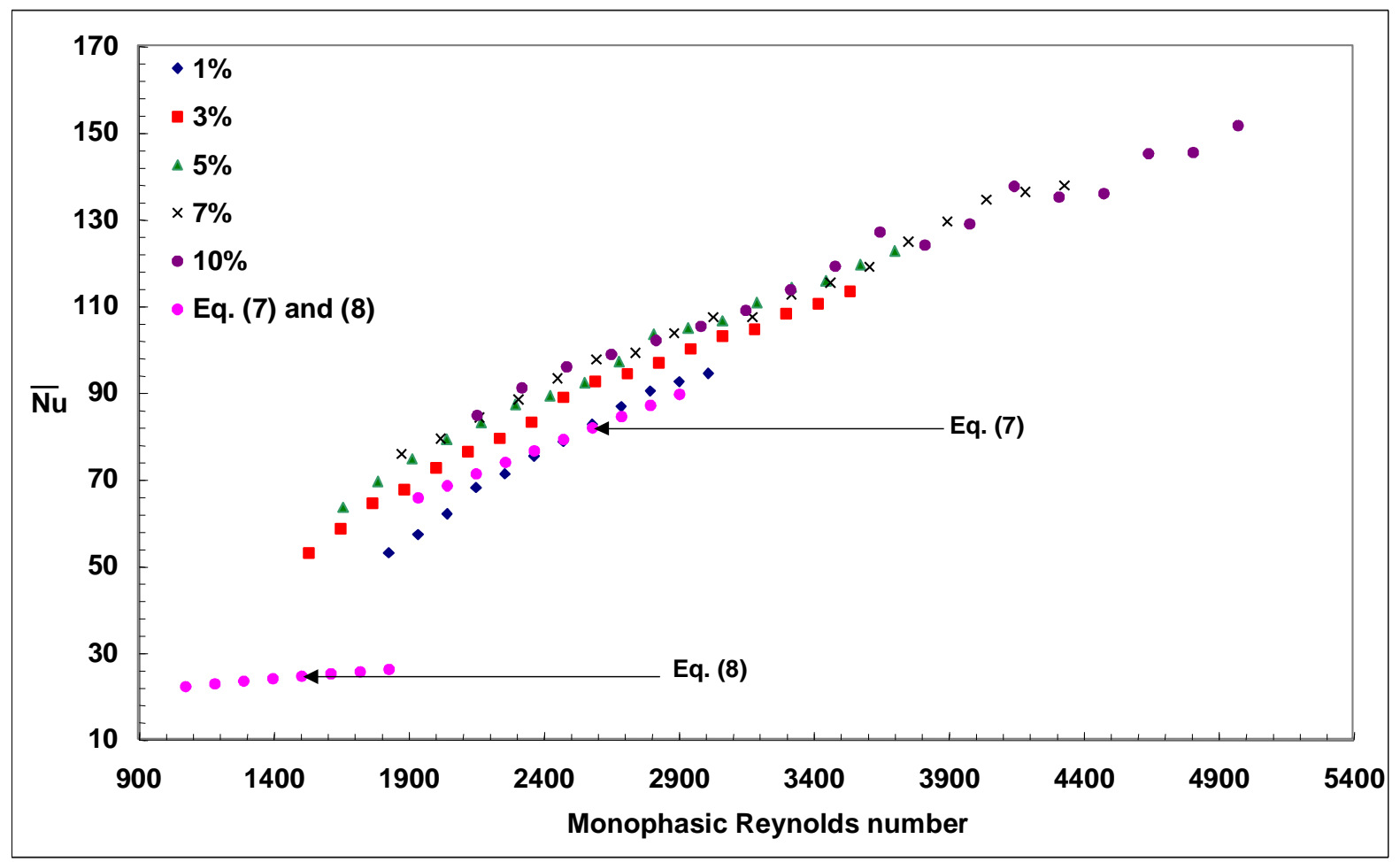

Figure 10b : Mean Nusselt number versus single phase Reynolds number for several volume fractions _ $\varphi_{\mathrm{w}}=15.000 \mathrm{~W} / \mathrm{m}^{2}$. 

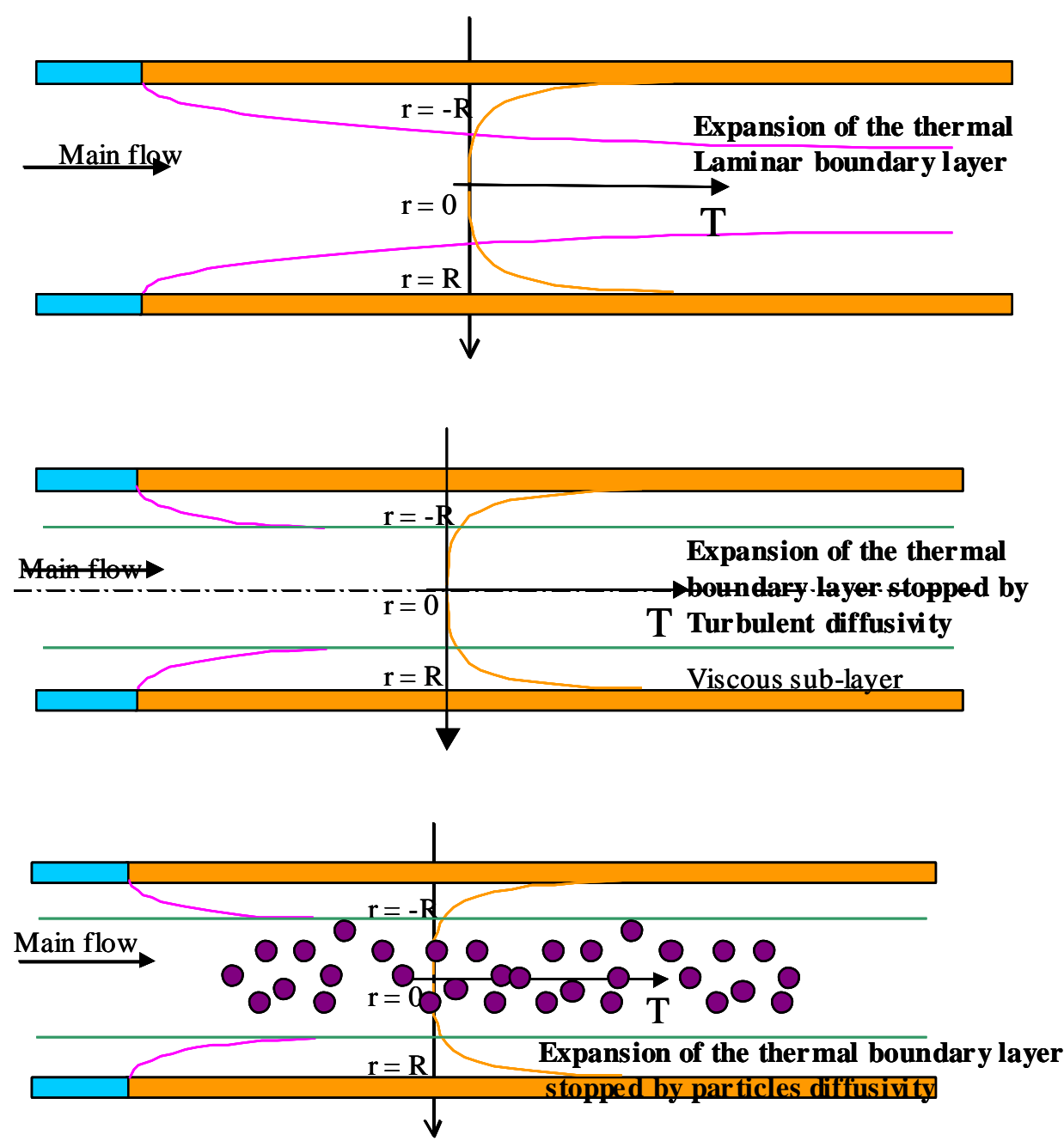

Figure 11: schema of the thermal mechanisms in solid-liquid flows 


\begin{tabular}{|c|c|c|c|}
\cline { 2 - 4 } \multicolumn{1}{c|}{} & $\rho\left(\mathrm{kg} \cdot \mathrm{m}^{-3}\right)$ & $\mathrm{k}\left(\mathrm{W} \cdot \mathrm{m}^{-1} \cdot \mathrm{K}^{-1}\right)$ & $\mathrm{Cp}\left(\mathrm{J} \cdot \mathrm{kg}^{-1} \cdot \mathrm{K}^{-1}\right)$ \\
\hline Water & 1000 & 0,6 & 4180 \\
Pure Glucosic solution & 1437,1 & 0,404 & 4180 \\
C.M.C & 888,25 & 0,6 & 4180 \\
Alginate particle & 1052,2 & - & - \\
\hline
\end{tabular}

Table 2: physical properties of each phases 